\title{
Lipocalin 2 Plays an Immunomodulatory Role and Has Detrimental Effects after Spinal Cord Injury
}

\author{
Khizr I. Rathore, ${ }^{1}$ Jennifer L. Berard, ${ }^{1}$ Adriana Redensek, ${ }^{1}$ Sabrina Chierzi, ${ }^{1}$ Ruben Lopez-Vales, ${ }^{1}$ Manuela Santos, ${ }^{2,3}$ \\ Shizuo Akira, ${ }^{4}$ and Samuel David ${ }^{1}$ \\ ${ }^{1}$ Center for Research in Neuroscience, The Research Institute of the McGill University Health Center, Montreal, Quebec H3G 1A4, Canada, ${ }^{2}$ Centre de \\ Recherche, Centre Hospitalier de l'Université de Montréal, Hôpital Nôtre-Dame, and ³Département de Médicine, Université de Montréal, Montréal, Québec \\ H2L 4M1, Canada, and ${ }^{4}$ Department of Host Defense, Osaka University, Osaka 565-0871, Japan
}

Lipocalin 2 (Lcn2) plays an important role in defense against bacterial infection by interfering with bacterial iron acquisition. Although Lcn2 is expressed in a number of aseptic inflammatory conditions, its role in these conditions remains unclear. We examined the expression and role of Lcn 2 after spinal cord injury (SCI) in adult mice by using a contusion injury model. Lcn 2 expression at the protein level is rapidly increased 12-fold at $1 \mathrm{~d}$ after SCI and decreases gradually thereafter, being three times as high as control levels at $21 \mathrm{~d}$ after injury. Lcn 2 expression is strongly induced after contusion injury in astrocytes, neurons, and neutrophils. The Lcn2 receptor (Lcn2R), which has been shown to influence cell survival, is also expressed after SCI in the same cell types. Lcn2-deficient $\left(L c n 2^{-/-}\right)$mice showed significantly better locomotor recovery after spinal cord contusion injury than wild-type $\left(\right.$ Lcn2 $\left.{ }^{+/+}\right)$mice. Histological assessments indicate improved neuronal and tissue survival and greater sparing of myelin in $L c n 2^{-/-}$mice after contusion injury. Flow cytometry showed a decrease in neutrophil influx and a small increase in the monocyte population in $L c n 2^{-/-}$injured spinal cords. This change was accompanied by a reduction in the expression of several pro-inflammatory chemokines and cytokines as well as inducible nitric oxide synthase early after SCI in $\mathrm{Lcn} 2^{-/-}$mice compared with wild-type animals. Our results, therefore, suggest a role for Lcn 2 in regulating inflammation in the injured spinal cord and that lack of Lcn2 reduces secondary damage and improves locomotor recovery after spinal cord contusion injury.

\section{Introduction}

Spinal cord injury (SCI) is associated with an inflammatory response that contributes to delayed secondary damage. Activated astrocytes, microglia, and neurons contribute to the initial inflammatory response by producing a number of pro-inflammatory cytokines and mediators (Bartholdi and Schwab, 1997; Yang et al., 2004, 2005; Pineau and Lacroix, 2007; Pineau et al., 2010) that are cytotoxic for neurons and glial cells at the lesion site. In addition, some of these molecules are important for recruiting immune cells to the lesion site and play a role in modulating the wound-healing response after SCI (Popovich and Longbrake, 2008).

Lipocalin 2 ( $\operatorname{Lcn} 2)$, also known as neutrophil gelatinaseassociated lipocalin (NGAL), is a siderophore-binding protein that is reported to play an important role in the innate immune

Received Jan. 7, 2011; revised July 5, 2011; accepted July 26, 2011.

Author contributions: K.I.R., M.S., and S.D. designed research; K.I.R., J.L.B., A.R., S.C., and R.L.-V. performed research; M.S. and S.A. contributed unpublished reagents/analytic tools; K.I.R., J.L.B., and S.C. analyzed data; K.I.R. and S.D. wrote the paper.

This work was supported by a grant from the Canadian Institutes of Health Research (CIHR) to S.D. M.S. was supported by the Natural Sciences and Engineering Research Council of Canada (Grant number 298518-06). K.I.R. was a recipient of a studentship from the CIHR Neuroinflammation Training Program. We thank Hiba Kazak and Ourania Tsatas for technical assistance and Margaret Attiwell for help with illustrations.

Correspondence should be addressed to Dr. Samuel David, Center for Research in Neuroscience, McGill University Health Center Research Institute, Livingston Hall, Room L7-210, 1650 Cedar Avenue, Montreal, QC H3G 1A4, Canada. E-mail:sam.david@mcgill.ca.

DOI:10.1523/JNEUROSCI.0116-11.2011

Copyright $\odot 2011$ the authors $\quad 0270-6474 / 11 / 3113412-08 \$ 15.00 / 0$ response to certain kinds of bacterial infection (Flo et al., 2004). Siderophores are bacterial molecules that bind iron and are internalized by a receptor-mediated mechanism. They allow bacteria to obtain iron, an element that is critical for their growth and survival, from within the host (Wandersman and Delepelaire, 2004). The biological functions of $\operatorname{Lcn} 2$ stem from its ability to bind iron-loaded siderophores (Goetz et al., 2002), thus depriving pathogenic bacteria of iron. Lcn 2 has also been shown to exert its effects on mammalian cells through its receptor Lcn2R (24p3R). Cells that expressed Lcn2R were sensitized to Lcn2triggered apoptosis through a mechanism dependent on the intracellular depletion of iron (Devireddy et al., 2005). Recent reports have suggested that $\operatorname{Lcn} 2$ may play a similar role in sensitizing microglia and astrocytes in vitro to cell death induced by cytotoxic agents such as nitric oxide (Lee et al., 2007, 2009). In the CNS, expression of Lcn 2 has been reported in the choroid plexus in response to peripheral lipopolysaccharide (LPS) administration (Marques et al., 2008), as well as in the striatum after intrastriatal injections of LPS (Glezer et al., 2007). Its expression was also reported to be upregulated in a microarray analysis of SCI tissue (De Biase et al., 2005). However, what role Lcn2 plays in CNS inflammation and injury remains unknown.

Here we report that $\mathrm{Lcn} 2$ plays a detrimental role in a mouse model of spinal contusion injury. Using $L \mathrm{cn} 2^{-1-}$ mice, we show that Lcn 2 contributes to inflammation and secondary damage in the injured spinal cord. Lcn $2^{-/-}$mice also show improved locomotor recovery as compared with wild-type controls. Our results 

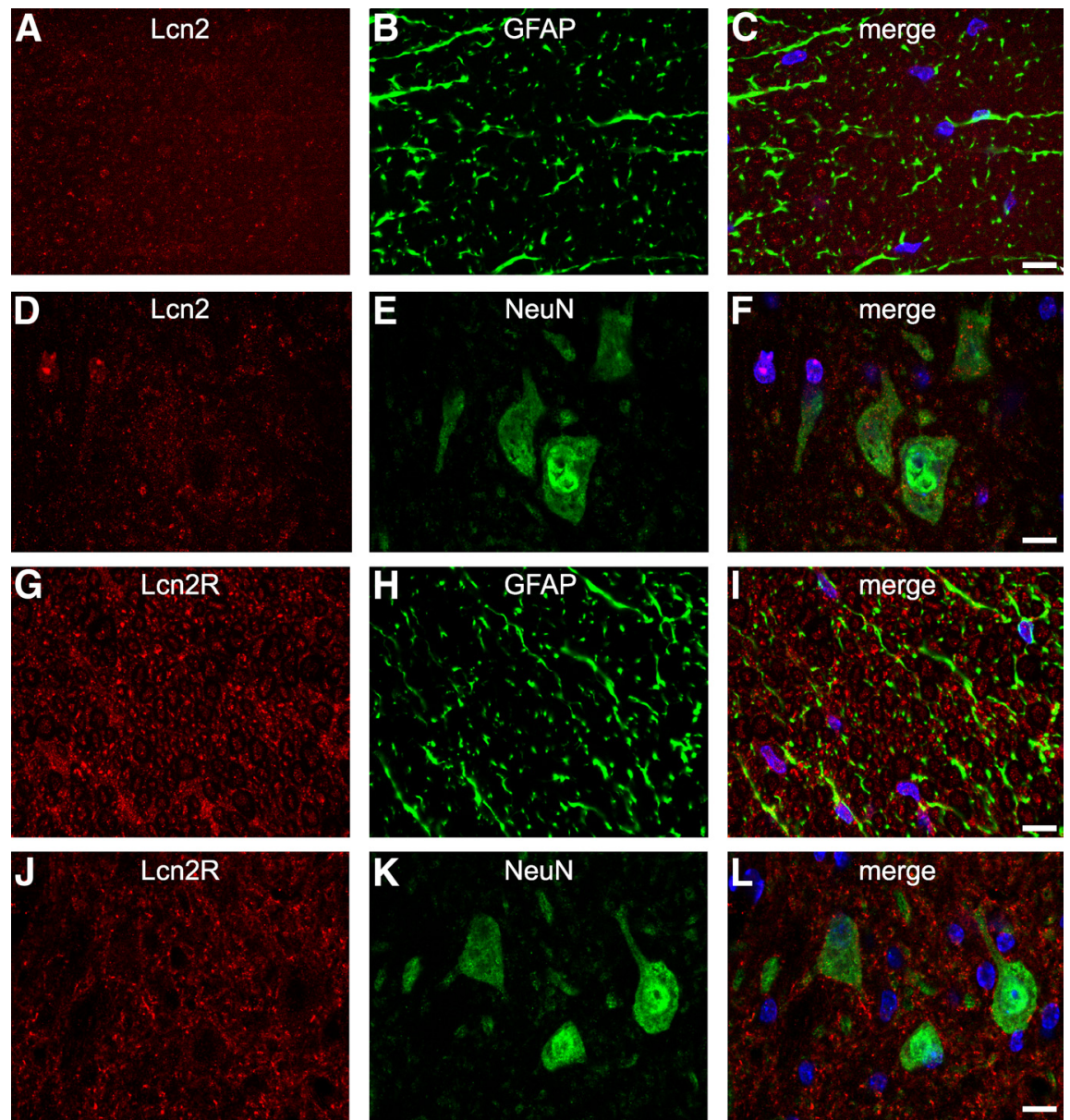

Figure 1. Lcn2 and Lcn2R expression in the uninjured spinal cord. Confocal images show double immunofluorescence labeling for $L \mathrm{cn} 2(\boldsymbol{A}-\boldsymbol{F})$ and $\operatorname{Lcn} 2 R(\boldsymbol{G}-\boldsymbol{L})$ in the uninjured spinal cord. Representative micrographs show extremely low levels of $L \mathrm{cn} 2$ and $L \mathrm{Ln} 2 \mathrm{R}$ in astrocytes and neurons in the uninjured spinal cord. Scale bars, $10 \mu \mathrm{m}$.

suggest that Lcn 2 plays a detrimental role after SCI, likely through the modulation of diverse pathways.

\section{Materials and Methods}

Animals. Lcn2 ${ }^{+/+}$and Lcn2 $2^{-/-}$mice on a C57BL/6 background (Flo et al., 2004; Huang et al., 2009) between 2 and 4 months of age were used for spinal cord contusion injury ( $60 \mathrm{kdyn}$ force, $500-700 \mu \mathrm{m}$ tissue displacement) using the Infinite Horizons impactor (Precision Scientific Instrumentation). Mice were deeply anesthetized with ketamine, xylazine, and acepromazine (100,50, and $3 \mathrm{mg} / \mathrm{kg}$ ), a single laminectomy was done, and the contusion injury was performed at the T10 thoracic level (Ghasemlou et al., 2005). Motor control was evaluated using the nine-point Basso Mouse Scale (BMS) (Basso et al., 2006) by two observers trained in the Basso laboratory at The Ohio State University. Consensus scores were then recorded. These analyses were performed by observers blinded to experimental groups. All procedures were approved by the McGill University Animal Care Committee and followed the guidelines of the Canadian Council on Animal Care.

Western blotting. A 5-mm-long piece of spinal cord centered at the lesion site was removed at $1,3,7,14,21$, and $28 \mathrm{~d}$ postinjury (dpi) $(n=$ 3 mice per group) after intracardiac perfusion with $10 \mathrm{ml}$ of $0.1 \mathrm{M}$ phosphate buffer. Total protein was extracted using radioimmunoprecipitation assay buffer containing 1\% Nonidet P-40 (Sigma), 1\% sodium deoxycholate (BDH Chemicals), $2 \%$ SDS, $0.15 \mathrm{~m}$ sodium phosphate, $\mathrm{pH}$ 7.2, 2 mм EDTA, and a mixture of protease inhibitors (Roche Diagnostics). Twenty micrograms of total protein were loaded onto a $4-12 \%$ SDS-PAGE (Novex Bis-Tris gel; Invitrogen) and then transferred to polyvinylidene fluoride membrane (Millipore). Membranes were blocked with 3\% nonfat milk in PBS-Tween 20 $(0.05 \%)$ and incubated at $4^{\circ} \mathrm{C}$ overnight with goat anti-mouse Lcn2 (1:400; R\&D Systems). Blots were washed and incubated with peroxidase-conjugated IgG (1:200,000; Jackson ImmunoResearch) and detected with Western Lightning Chemiluminescence Reagent Plus (PerkinElmer Life and Analytical Sciences). Blots were subsequently reprobed with rabbit anti- $\beta$-actin (1:400; SigmaAldrich) to confirm equal loading. Experiments were done in triplicate.

Quantitative real-time reverse transcriptionPCR. Approximately $5 \mathrm{~mm}$ of spinal cord tissue containing the lesion site was removed at 1 , 3, 7, and 21 dpi. Total RNA was isolated from SCI tissue as described previously (Rathore et al., 2008) using the RNeasy Lipid Tissue kit (Qiagen), and quantitative real-time reverse transcription (QRT)-PCR was performed using the MX4000 (Stratagene) as per the manufacturer's protocol for all mRNA expression analyses. The relative expression between the samples was calculated based on the threshold cycle $\left(C_{\mathrm{T}}\right)$ value, and was standardized to the amount of a housekeeping gene, glyceraldehyde 3-phosphate dehydrogenase (GAPDH), using the $\Delta C_{\mathrm{T}}$ method (Livak and Schmittgen, 2001): $\Delta \Delta C_{\mathrm{T}}=\left(C_{\mathrm{T}, \text { Target }}-C_{\mathrm{T}, \mathrm{GAPDH}}\right)-\left(C_{\mathrm{T}, \text { Target }}-\right.$ $\left.C_{\mathrm{T}, \mathrm{GAPDH}}\right)_{\text {(calibrator avg) }}$. Results are shown as the mean (fold increase over uninjured controls) \pm SEM, $n=4$.

Flow cytometry. Immune cells from the injured spinal cord were analyzed by flow cytometry at 2 dpi as described previously (Stirling and Yong, 2008). Briefly, the spinal cord samples were mechanically disrupted using a Dounce homogenizer and passed through an $80 \mu \mathrm{m}$ cell strainer (BD Falcon). Cells were then collected by mild centrifugation and resuspended in PBS containing $1 \%$ fetal bovine serum. Samples were divided, and cells alone and isotype-matched control samples were generated to control for nonspecific binding of antibodies and for autofluorescence. Isotype control antibodies were purchased from BD Biosciences PharMingen and included phycoerythrin (PE)-labeled rat IgG2b, allophycocyanin (APC)labeled rat IgG2b, and peridinin chlorophyll-a protein (PerCP)-conjugated rat IgG2b. After cell counts, the samples were Fc blocked (BD Biosciences) to minimize background staining. The following antibodies were purchased from BD Biosciences PharMingen and were optimized and used to determine different populations of resident microglia and blood-derived leukocytes: APC-conjugated rat anti-mouse CD45 (leukocyte common antigen, Ly-5), PE-conjugated rat anti-mouse Ly-6G and Ly-6C (Gr-1), and PerCPconjugated rat anti-mouse $\mathrm{CD} 11 \mathrm{~b}$. After $30 \mathrm{~min}$ of incubation with combinations of antibodies at $4^{\circ} \mathrm{C}$, the samples were washed and fixed in $1 \%$ paraformaldehyde. The samples were then immediately analyzed with a FACSCalibur Flow Cytometer (BD Biosciences). Equal numbers of cells were analyzed for all conditions. Density plots were created using CellQuest software (BD Biosciences), and cells alone and isotype controls were used to set quadrants. CD45-positive gates ensured that only leukocytes and resident microglia were included in the analysis. The proportions of blood-derived leukocytes (neutrophils and monocytes) $\left(\mathrm{CD} 45^{\text {high }}\right.$ :CD11b), neutrophils $\left(\mathrm{CD} 45^{\text {high }}: \mathrm{Gr}-1^{\text {high }}\right)$, and resident microglia (CD45 $\left.{ }^{\text {low }}: \mathrm{CD} 11 \mathrm{~b}\right)$ were determined from density plots (CD45, $y$-axis; CD11b or Gr- $1, x$-axis) using CellQuest software and compared between the different groups $(n=4)$.

Immunofluorescence and histological analyses. Animals were perfused with $0.1 \mathrm{~m}$ phosphate buffer, followed by $4 \%$ paraformaldehyde in $0.1 \mathrm{M}$ phosphate buffer, pH 7.2. A $7 \mathrm{~mm}$ length of spinal cord tissue centered on the lesion epicenter was dissected out and cryoprotected, and $12-\mu \mathrm{m}$-thick cryostat sections were obtained. Tissue sections were incubated with $0.1 \mathrm{~m}$ PBS 
containing $2 \%$ normal goat serum and $1 \%$ ovalbumin to block nonspecific binding of antibodies. Tissue sections were incubated overnight at $4^{\circ} \mathrm{C}$ with primary antibodies at the following concentrations: goat anti-Lcn2 (1:200; R\&D systems), rabbit anti-Lcn2R (1:100; Sigma/Atlas Antibodies), rat anti-glial fibrillary acidic protein (GFAP) (1:800; Invitrogen), rabbit anti-Ibal (1: 500; Wako Chemicals), and rat anti-7/4 (1:500; Serotec) at room temperature. After washing, sections were then incubated for $1 \mathrm{~h}$ at room temperature with Alexa-568-conjugated donkey anti-rabbit IgG (1:200; Invitrogen), Alexa-488conjugated donkey anti-rat IgG (1:200; Invitrogen), or Alexa-568-conjugated donkey anti-goat (1:200; Invitrogen). Nuclear staining for confocal analysis was done by incubating sections with TOPRO-3 (1:5000, Invitrogen). Sections were mounted with Slowfade Gold antifade media (Invitrogen), and a coverslip was applied. For confocal microscopy, immunostained sections were viewed with a spinning disk confocal system (PerkinElmer) connected to an EclipseTE2000 (Nikon). Image capture and analysis were conducted with MetaMorph (Molecular Devices). For a negative control for Lcn2 antibody, we stained spinal cord section from Lcn 2 knock-out mice. No staining was detected, as shown in Results. The Lnc2 receptor antibody was generated against a 60-mer recombinant protein fragment of Lnc2R. The antibody was validated using an antigen microarray against 384 different antigens as well as by immunostaining by the company that generated the antibody (Atlas Antibodies). We were, however, unable to obtain the recombinant protein for immunodepletion, and there are no knockout mice available.

To detect ventral horn neurons, tissue sections were stained by incubating tissue in $0.1 \%$ cresyl violet (Sigma-Aldrich) for $10 \mathrm{~min}$ or in $0.1 \%$ Luxol fast blue overnight and cleared in $0.05 \%$ lithium carbonate to stain for myelin. This was followed by dehydration through ascending alcohols and Hemo-De (Thermo Fisher Scientific). All bright-field and immunofluorescent microscopy was conducted on the Axioskop 2 Plus (Zeiss), by using a QImaging Retiga 1300 camera and BIOQUANT image analysis system for image capture and analysis. For the analysis of the GFAP-positive area in the spinal cord cross sections, the pixel density function of the BIOQUANT image analysis system was used to analyze the total cross-sectional area in stained sections. For assessment of myelin sparing, the total extent of myelin staining in the tissue section was quantified using the pixel count function in BIOQUANT and normalized to the pixel count for the entire cross section. Spared myelin was presented as a percentage of myelin at the lesion site compared with the percentage of myelin in intact spinal cord.

\section{Results}

Expression of Lcn 2 is markedly upregulated after SCI

We assessed the expression of Lcn 2 in the uninjured spinal cord by double immu-
A

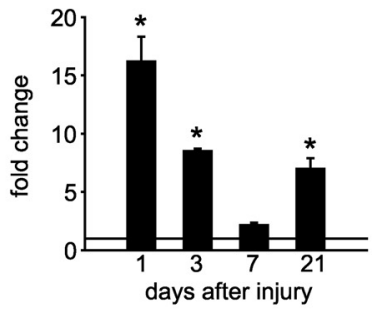

B

ui $\quad 1 d \quad 3 d \quad 7 d \quad 14 d \quad 21 d \quad 28 d$

$\operatorname{Lcn} 2$

$\beta$-actin

D
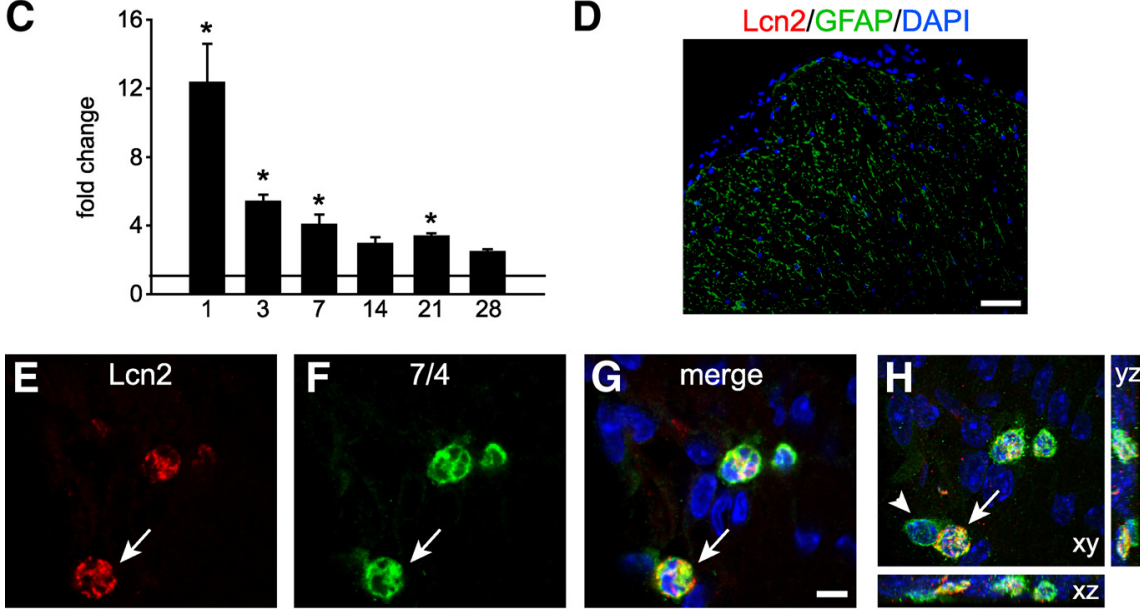

Lcn2
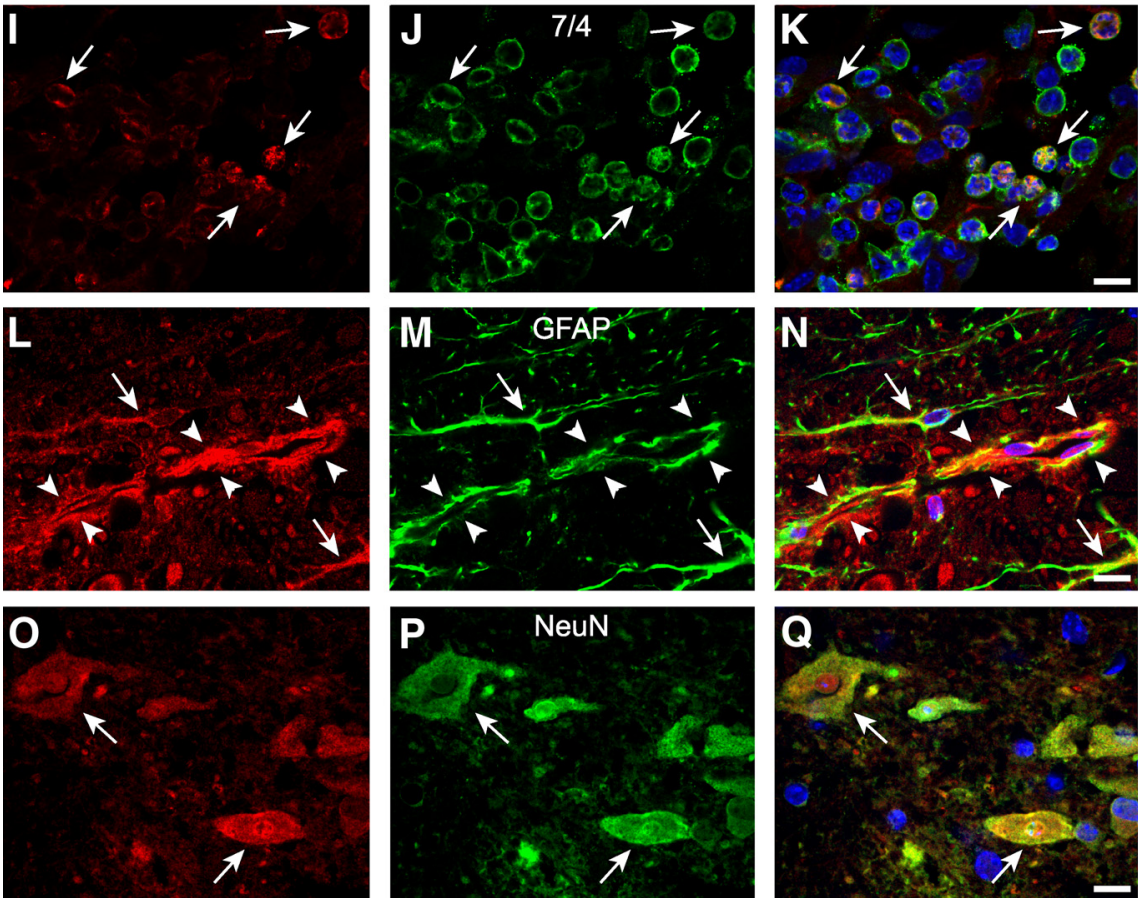

Figure 2. Len2 expression after SCl. $A$, Changes in Lcn $2 \mathrm{mRNA}$ expression as determined by QRT-PCRin the injured spinal cord at various times after injury. Lcn2 mRNA levels are significantly upregulated above controls at 1,3, and $21 \mathrm{dpi}\left({ }^{*} p<0.05,2\right.$-sample unequal variance $t$ test, $n=3$ for each time point). $\boldsymbol{B}$, Representative Western blot showing $L \mathrm{cn} 2$ protein in spinal cord homogenates from uninjured naive (ui) mice and at several time points after $\mathrm{SCl}$. $\beta$-Actin was used as a loading control. Note the marked increased immediately after $\mathrm{SCl}$ and its gradual decrease thereafter. C, Densitometric quantification of Western blot data ${ }^{*} p<0.05,2$-way ANOVA, posthoc Tukey; $n=3$ per group). $\boldsymbol{D}$, Negative staining control shows no anti-Lcn2 labeling (red) in $L\left(\mathrm{Cn} 2^{-1-}\right.$ spinal cord tissue, double labeled with anti-GFAP (green); nuclei are stained with DAPI. E-G, Single-plane confocal images ( $0.6 \mu \mathrm{m}$ thickness) of double immunofluorescence labeling of spinal cord tissue sections $1 \mathrm{~d}$ after SCI stained for Lcn2 (red) and 7/4 for neutrophils (green); arrows indicate double-labeled cells. $\boldsymbol{H}, \mathrm{A}$ ful $z$-stack image $(14 \mu \mathrm{m})$ showing double labeling for $L n c 2$ and $7 / 4$ in the $x-y, x-Z$, and $y-z$ planes. $I-\boldsymbol{K}$, Single-plane confocal images showing double immunofluorescence labeling of infiltrating 7/4-positive neutrophils expressing $L$ Ln 2 (arrows) in the injured spinal cord at 3 dpi. Similar confocal images showing that GFAP-positive astrocytes $(\boldsymbol{L}-\boldsymbol{N})$ as well as NeuN-positive neurons $(\mathbf{O}-\mathbf{Q})$ also express $\mathbf{L}$ (cn2 at 1 dpi (arrows indicate double-labeled cells; arrowheads point to a longitudinal profile of a blood vessel that shows Lnc2 labeling of the endothelial cells lining the inner aspect of the GFAP staining). Scale bars: $\boldsymbol{D}, 50 \mu \mathrm{m} ; \boldsymbol{E}-\mathbf{G}, 5 \mu \mathrm{m} ; \boldsymbol{I}-\mathbf{Q}, 10 \mu \mathrm{m}$. 


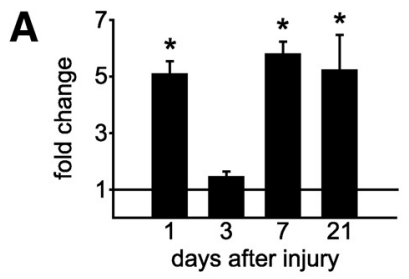

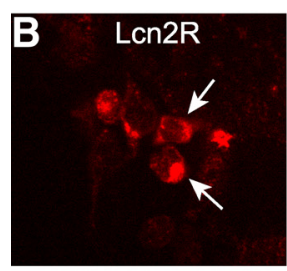
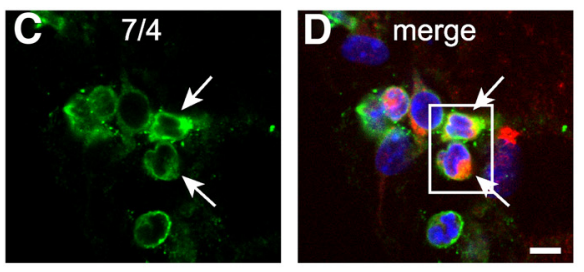

Lcn2R
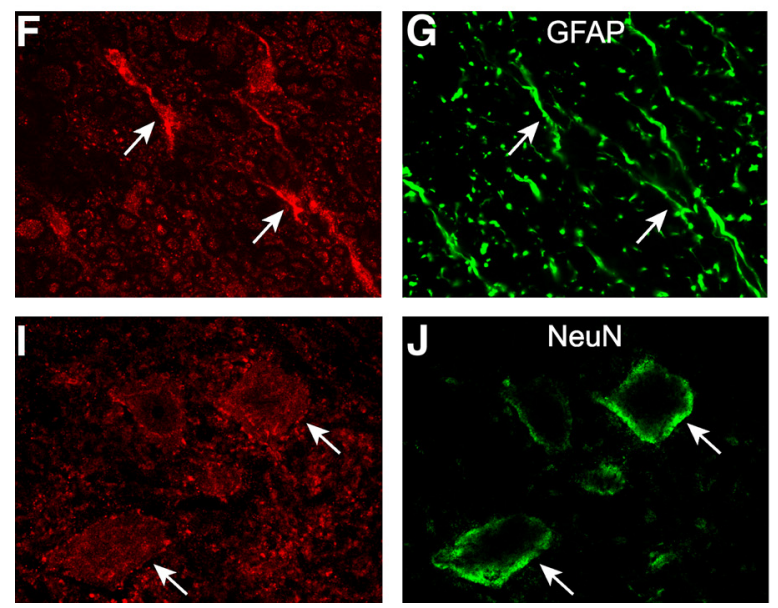

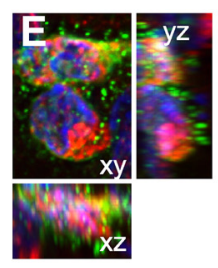

merge
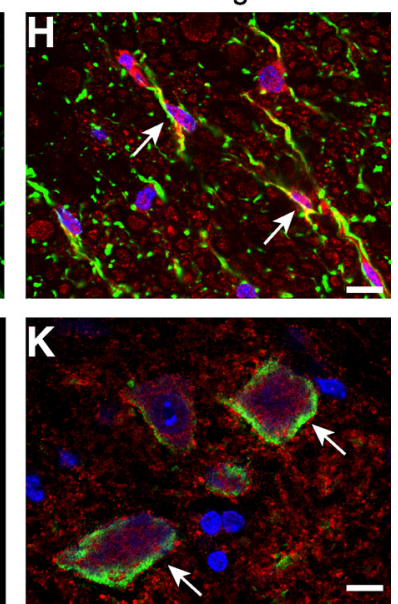

Figure 3. Lcn2 receptor expression after SCl. $\boldsymbol{A}$, Graph showing changes in Len $2 R$ mRNA expression as detected by QRT-PCR. Expression levels are increased at 1, 7, and $21 \mathrm{dpi}\left({ }^{*} p \leq 0.05 ; 2\right.$-way ANOVA, post hoc Tukey). $\boldsymbol{B}-\boldsymbol{D}$, Single-plane confocal images (0.6 $\mu \mathrm{m}$ thickness) showing double labeling for $\mathrm{Lcn} 2 \mathrm{R}$ and $7 / 4$ (arrows indicate double-labeled cells) at $1 \mathrm{dpi}$. $\boldsymbol{E}, \mathrm{A}$ full $z$-stack image (14 $\mu \mathrm{m}$ ) of the area in the box in $\boldsymbol{D}$ showing double labeling for $\operatorname{Lnc} 2 R$ and $7 / 4$ in the $x-y, x-z$, and $y-z$ planes. $\boldsymbol{F}-\boldsymbol{K}$, Single-plane confocal images showing GFAP-positive astrocytes (arrows) and NeuN-positive neurons at 1 dpi. Scale bars: $\boldsymbol{B}-\boldsymbol{D}, 5$ $\mu \mathrm{m} ; \boldsymbol{E}-\boldsymbol{J}, 10 \mu \mathrm{m}$.

nofluorescence labeling and confocal microscopy. There was minimal Lcn2 staining in the uninjured spinal cord (Fig. 1A$F)$. After SCI, Lcn 2 mRNA was significantly induced $\sim 16$-fold higher than control levels at $1 \mathrm{~d}$ after contusion injury as determined by QRT-PCR (Fig. 2A). The mRNA expression levels progressively decreased from 3 through $7 \mathrm{dpi}$, reaching levels approximately twofold higher than control levels at day 7 , and increasing to levels approximately sevenfold higher than control levels at 21 dpi. Densitometric quantification of Western blot analysis showed a 12 -fold increase in Lcn2 protein at $1 \mathrm{dpi}$ and a gradual decrease thereafter, but the levels still remained at approximately threefold higher than control levels at day 21 (Fig. $2 B, C)$. We next assessed the cellular localization of $\mathrm{Lcn} 2$ after SCI using double immunofluorescence labeling and confocal microscopy. Double immunofluorescence staining at 1 dpi showed that 7/4-positive neutrophils close to the lesion site expressed Lcn2 (Fig. $2 E-H$ ). Confocal single-plane images showed Lcn2 expression in 7/4-positive neutrophils at $1 \mathrm{dpi}($ Fig. $2 E-G$ ). Colocalization is seen further in the complete $z$-stack images with $x-y, x-z$, and $y-z$ planes (Fig. $2 H$ ). Single-plane images from 3 dpi tissue also showed expression of Lcn2 in neutrophils at this time point (Fig. $2 I-K)$. Single-plane images from 1 dpi also showed Lcn 2 expression in GFAP-positive astrocytes (Fig. $2 L-N$ ). Neurons in the ventral gray matter of the injured spinal cord were also positive for Lcn2

(Fig. 2O-Q). At $1 \mathrm{dpi}$, elongated profiles that are likely to be endothelial cells were also positive for Lcn2 (Fig. $2 L-N$, arrowheads). Lcn2 was not detected in oligodendrocytes or Ibal-positive microglia (data not shown).

We next assessed the expression of Lcn2R, also known as BOCT (brain-type organic cation transporter), after contusion injury. Lcn2R levels are low in the uninjured spinal cord as determined by double immunofluorescence labeling and confocal microscopy (Fig. 1G-L). The mRNA expression of the Lcn2 receptor as determined by QRT-PCR is significantly increased in spinal cord tissue after contusion (Fig. 3A). Immunofluorescence labeling showed that after SCI, Lcn2R was expressed in neutrophils (Fig. $3 B-D$ ), astrocytes (Fig. $3 E-G$ ), and weakly in neurons (Fig. $3 H-J$ ).

\section{Lcn2 deletion improves locomotor recovery after SCI}

The effects of Lcn2 deficiency on locomotor recovery after SCI were assessed using the BMS (Basso et al., 2006). The BMS analysis is specifically designed to assess open-field locomotor recovery in mice after contusion injury. This analysis shows that $\mathrm{Lcn}^{-/-}$mice had significantly better recovery of locomotor function than did wild-type mice starting at day 5 after injury (Fig. 4A). By 10 dpi, $L c n 2^{-1-}$ mice had an average score of $4.05 \pm 0.43$, which corresponds to occasional stepping, whereas $\mathrm{Lcn} 2^{+/+}$mice had an average score of $3.0 \pm 0.61$, which corresponds to paw placement, but no stepping. By $28 \mathrm{dpi}, \mathrm{Lcn} 2^{-1-}$ had an average score of $4.9 \pm 0.39$, whereas $\mathrm{Lcn} 2^{+/+}$animals had an average score of $3.7 \pm 0.36$. $\mathrm{Lcn} 2^{-/-}$mice also had significantly better improvement of finer aspects of locomotor control as reflected by higher BMS subscores than $\mathrm{Lcn} 2^{+/+}$mice. BMS subscores can only be scored in mice that are frequently stepping and attain a score of 5 . Accordingly, wild-type mice had a subscore of zero, while the Lcn2-null mice had an average subscore of $1.2 \pm 0.29$ (SEM) at 28 dpi.

\section{$L c n 2^{-/-}$mice have increased neuron and tissue sparing} after SCI

Given the proposed role for Lcn2 in promoting cell death, we assessed neuronal survival in the ventral horn of $\mathrm{Lcn2}^{-/-}$and $\mathrm{Lcn} 2^{+/+}$mice at $28 \mathrm{dpi}$. Quantification of neurons in cresyl violet-stained tissue sections showed that $L \mathrm{cn} 2^{-1-}$ mice have significantly more neurons at 500 and $1000 \mu \mathrm{m}$ rostral and $1000 \mu \mathrm{m}$ caudal to the injury site (Fig. $4 B-D$ ). Using GFAP staining as a marker to label astrocytes, we examined cross sections of the spinal cord at the epicenter and $200 \mu \mathrm{m}$ rostral and caudal to the lesion site at 3 and $7 \mathrm{dpi}$. Our results show that there is significantly increased tissue sparing in $\mathrm{Lcn} 2^{-/-}$mice as compared with wild-type injured controls at 3 and $7 \mathrm{dpi}$. The data for the $7 \mathrm{dpi}$ are shown in Figure 5, $A$ and $B$. Similar results were seen at 3 dpi (data not shown). We also assessed myelin sparing at the lesion 

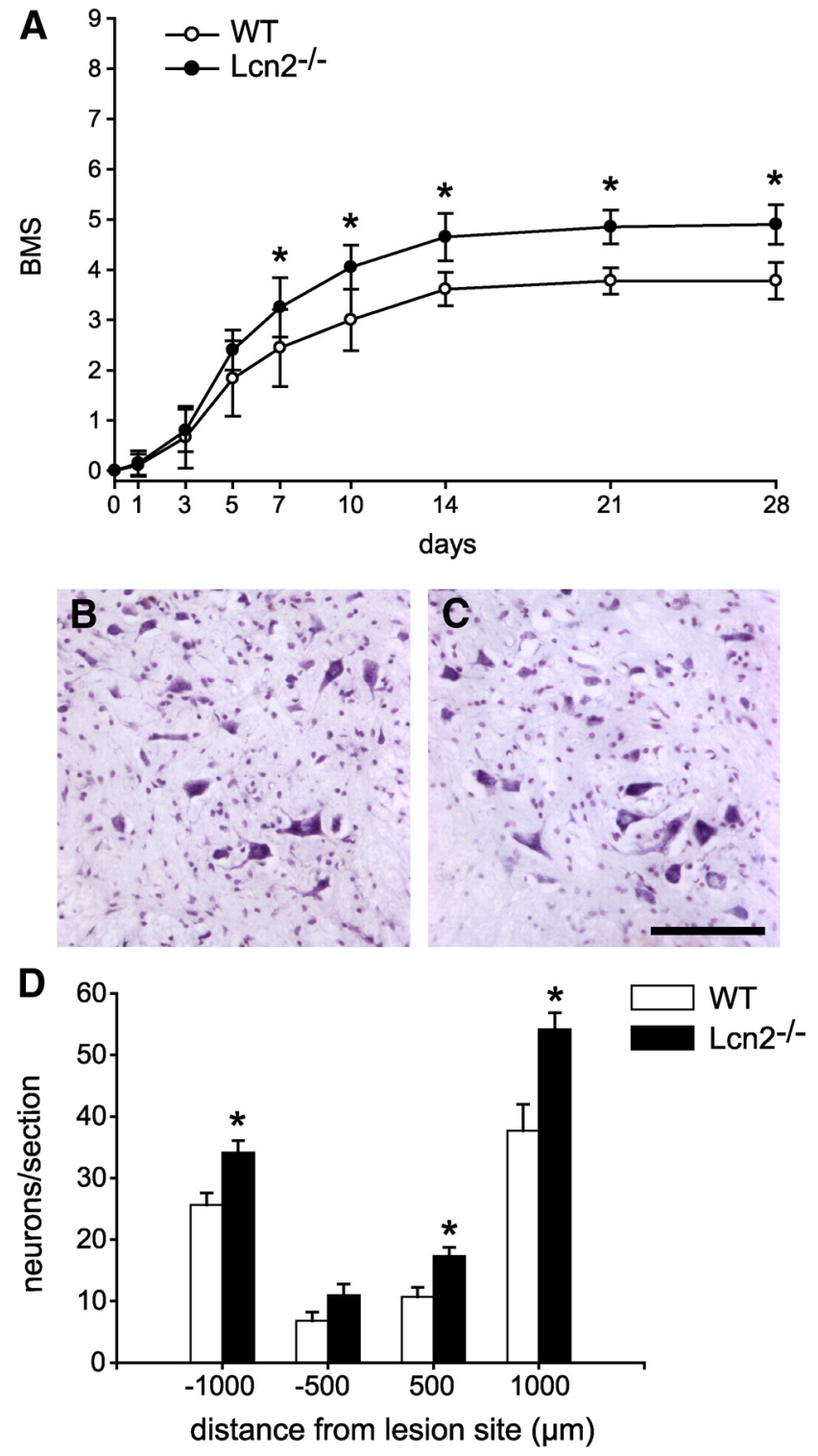

Figure 4. Improved locomotor recovery and neuronal sparing in $L \mathrm{cn} 2^{-1-}$ mice. A, Graph showing the BMS score of injured wild-type (WT) and $L \mathrm{Cn} 2^{-/-}$after SCI. $\mathrm{Lcn} 2^{-/-}$mice have significantly improved locomotor recovery from 5 dpi onwards, mean \pm SD. $n=9$ for WT, $n=$ 10 for $\mathrm{LCn}^{-/-}{ }^{*}{ }^{*} p<0.05$, two-way repeated-measures (RM)-ANOVA, post hoc Tukey. B, C, Micrographs of cresyl violet-stained sections of the spinal cord $1000 \mu \mathrm{m}$ caudal to the lesion showing the ventral horn region of wild-type $(\boldsymbol{B})$ and $L \mathrm{Cn} 2^{-1-}(\boldsymbol{C})$ mice. Note the greater number of surviving large neurons in the $L \mathrm{Cn} 2^{-/-}$mouse. $D$, Quantification of neuronal survival at 500 and $1000 \mu \mathrm{m}$ distances rostral and caudal to the lesion site, mean \pm SEM. ${ }^{*} p<$ 0.05 , two-tailed $t$ test, $n=6$ for each genotype. Scale bar, $100 \mu \mathrm{m}$.

site using Luxol fast blue staining of tissue sections. $\mathrm{Lcn2} 2^{-/-}$mice $(n=6)$ showed a significantly higher percentage of myelin sparing $(52.9 \pm 4 \%)$ at the lesion epicenter than $L c n 2^{+/+}$animals $(28.4 \pm 4 \% ; n=4)(p \leq 0.05)$.

\section{$\operatorname{Lcn} 2^{-/-}$mice have altered cytokine expression and leukocyte infiltration}

We next assessed the mRNA expression of MIP1- $\alpha$, MCP-1, TNF- $\alpha$, IL- $1 \alpha$, IL- $1 \beta$, IL- 6 , and iNOS in the contused spinal cords of $\mathrm{Lcn} 2^{+/+}$and $\mathrm{Lcn} 2^{-/-}$mice at $1 \mathrm{dpi}$. The above chemokines and cytokines are reported to be expressed after SCI and contribute to the pro-inflammatory milieu that is present after SCI (Pineau and Lacroix, 2007). The relative mRNA expression of all these pro-inflammatory mediators was significantly reduced in Lcn $2^{-1-}$ mice (Fig. 6). Flow cytometry was used to quantify the infiltration of immune cells at the lesion site. Our expression data mentioned above indicate that Lcn2 and its receptor are expressed in cells infiltrating into the spinal cord at 1 and $3 \mathrm{dpi}$, a time when the majority of immune cells are neutrophils and monocytes (Stirling and Yong, 2008). We therefore assessed whether neutrophil/monocyte infiltration was altered in $\mathrm{Lcn} 2^{-/-}$mice at $2 \mathrm{dpi}$. CD45 was used as a marker for peripheral immune cells and microglia to sort cells obtained from injured spinal cords from $\mathrm{Lcn} 2^{-/-}$and $\mathrm{Lcn} 2^{+/+}$. Further gating with CD11b made it possible to distinguish between leukocytes $\left(\mathrm{CD} 45^{\text {high }} \mathrm{CD} 11 \mathrm{~b}^{+}\right)$and microglia $\left(\mathrm{CD} 45^{\text {low }} \mathrm{CD} 11 \mathrm{~b}^{+}\right)$. The leukocyte population was selected for further analysis and gated on Gr-1, a marker for both monocytes and neutrophils (Stirling et al., 2009). We detected two distinct populations of (CD $45^{\text {high }}$ Gr- $1^{+}$) cells, a Gr-1 ${ }^{\text {high }}$ population that corresponds to neutrophils (Fig. 7C,F, R8), and a Gr-1 ${ }^{\text {low }}$ population that corresponds to monocytes (Fig. 7C,F, R9). Our results show that, at 2 dpi, there are significantly fewer neutrophils in the injured spinal cord of $\mathrm{Lcn} 2^{-1-}$ mice $(49.6 \pm 0.97 \%$ of the leukocyte population) than in $L c n 2^{+/+}$mice $(59.5 \pm 0.86 \%$ of the leukocyte population). In addition, there is a small but statistically significant increase in the monocyte population in $\mathrm{Lcn} 2^{-/-}$mice to $30.3 \pm$ $0.82 \%$ compared with $25.1 \pm 0.51 \%$ in $L c n 2^{+/+}$mice. These results suggest that Lcn 2 may play a role in leukocyte infiltration into the injured spinal cord.

\section{Discussion}

Lcn2 is a siderophore-binding protein that is thought to be involved in several inflammatory pathologies in non-CNS tissues (Bolignano et al., 2010). We showed that Lcn2 plays a detrimental role in SCI in mice. Lcn 2 and its receptor are expressed in astrocytes, neurons, and infiltrating neutrophils after SCI. Lcn $2^{-1-}$ mice have improved locomotor recovery after SCI and increased neuron and tissue sparing. In addition, our results show that $\mathrm{Lcn} 2^{-/-}$mice have decreased expression of certain pro-inflammatory mediators and altered neutrophil/monocyte infiltration into the injured spinal cord.

The role of $\operatorname{Lcn} 2$ as part of the innate immune system defense against bacteria and pathogens is becoming better defined (Flo et al., 2004; Saiga et al., 2008; Chan et al., 2009; Nairz et al., 2009). However, its role in other inflammatory diseases and traumatic injuries has not been thoroughly examined. Our results suggest that, in the context of SCI, the observed increased expression of $\mathrm{Lcn} 2$ is likely to be detrimental. The Lcn 2 receptor is expressed on neurons in the injured spinal cord, suggesting that they may be sensitive to Lcn2-mediated cell death, as has been shown for other cells (Devireddy et al., 2005). Such a role for Lcn 2 in neuronal loss would be consistent with our observation of increased neuronal sparing in $L c n 2^{-1-}$ mice after SCI. Our results also show that Lcn 2 and its receptor are expressed in infiltrating leukocytes. The human form of Lcn 2 was first identified as neutrophil gelatinaseassociated lipocalin (NGAL), so called because it covalently binds human gelatinase B (MMP9), stabilizes it, and promotes its proteolytic activity (Triebel et al., 1992). Whether murine Lcn2 plays a similar role is disputed (Kjeldsen et al., 2000; Tveita et al., 2008; Berger et al., 2010). However, our own zymogram analysis of MMP9 activity after SCI showed no difference between $\mathrm{Lcn} 2^{-1-}$ and $L c n 2^{+1+}$ mice (data not shown). Our results indicate that there is a significant reduction in neutrophils in the spinal cord after SCI in Lcn $2^{-1-}$ mice, which may contribute to the improved outcome. The role of neutrophils in SCI is debated; several studies 


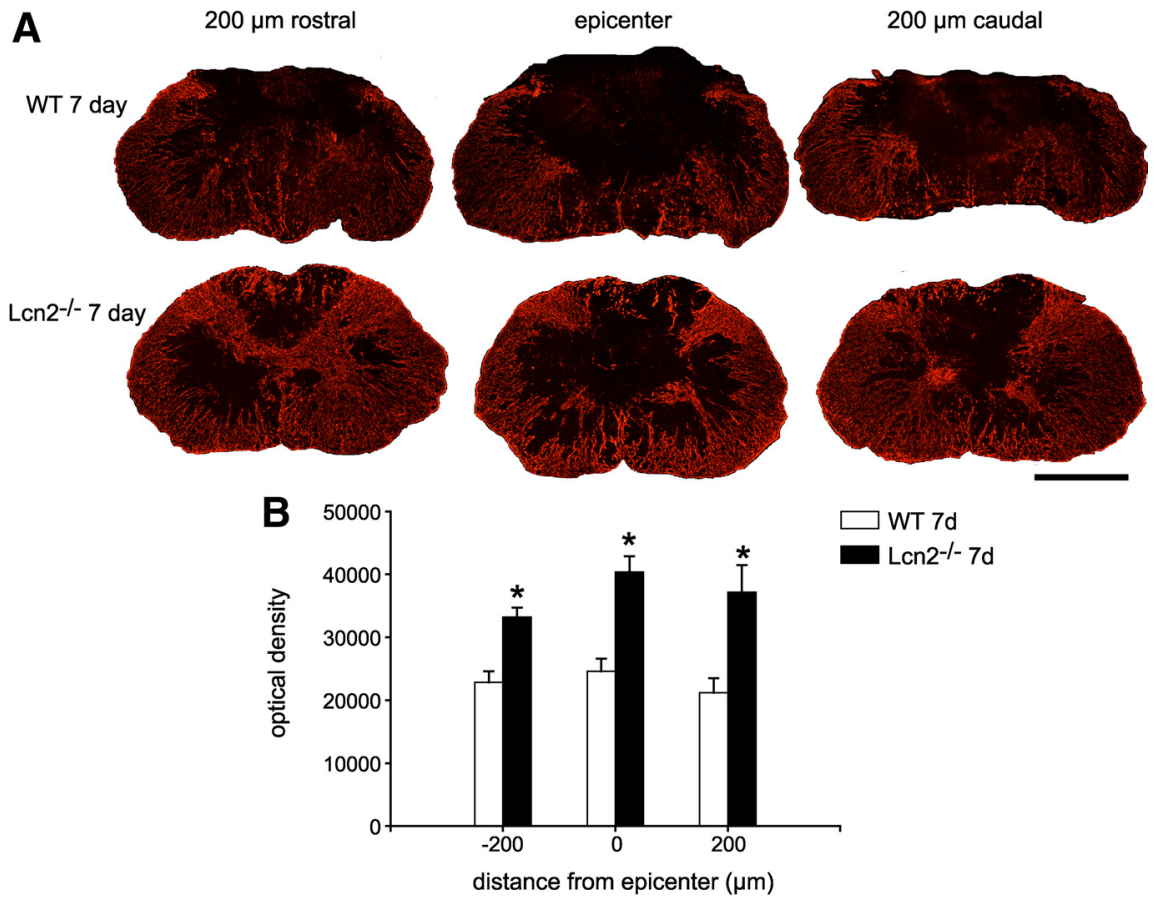

Figure 5. Increased tissue sparing in $L \mathrm{Cn} 2^{-1-}$ mice after $\mathrm{SCl}$. $\boldsymbol{A}$, Immunofluorescence labeling for GFAP in cross sections of the spinal cord of wild-type (WT) and $L \mathrm{cn} 2^{-1-}$ mice at $7 \mathrm{dpi}$. Note the greater degree of tissue sparing in the $L \mathrm{cn} 2^{-1-}$ spinal cords than in the wild-type mice. Representative micrographs from the lesion epicenter and at $200 \mu \mathrm{m}$ rostral and caudal are shown. $\boldsymbol{B}$, Quantification of GFAP immunofluorescence staining in entire cross section shows significantly greater tissue sparing in $L \mathrm{cn} 2^{-1-}$ mice than in wild-type control mice. Data are shown as mean \pm SEM. ${ }^{*} p<0.05$, two-tailed $t$ test, $n=3$ each genotype. Scale bar, $500 \mu \mathrm{m}$.

have suggested that neutrophils play a detrimental role after SCI (Taoka et al., 1997; Gris et al., 2004), while a recent report suggests that they may be beneficial after SCI (Stirling et al., 2009) through effects on wound healing and repair. Neutrophils have been shown to be largely insensitive to Lcn2-mediated death in vitro (Miharada et al., 2005), so the decrease in neutrophil numbers that we observed in the injured spinal cord in $L c n 2^{-1-}$ mice may be due to either direct or indirect effects of $\operatorname{Lcn} 2$ on neutrophil recruitment. We also detected a small but significant increase in monocyte infiltration into the injured spinal cord in $L c n 2^{-1-}$ mice. Whether this reflects an influx of a nonphlogistic (noninflammatory) type of monocytes, as has been reported under conditions in which there is resolution of inflammation (Serhan et al., 2008), is not yet known. A recent report has also suggested that certain monocyte populations can have beneficial effects on the injured spinal cord (Shechter et al., 2009). Consistent with these findings, we also observed a significant reduction in the expression of various pro-inflammatory mediators (MIP- $1 \alpha$, MCP-1, IL- $1 \alpha$, IL- $1 \beta$, TNF- $\alpha$, and iNOS) in the injured spinal cord of $L \mathrm{cn} 2^{-1-}$ mice. Together, these data suggest that $\operatorname{Lcn} 2$ may play a role in the initiation of inflammation and leukocyte migration into the injured spinal cord. Whether this is a direct effect of $\operatorname{Lcn} 2$ on leukocyte migration or a secondary effect of decreased cytokine synthesis will require further study.

As yet, the mechanism of Lcn2 function is thought to stem from its ability to bind iron. Len 2 cannot bind iron directly, but binds to siderophores that in turn bind iron. There are several bacterial siderophores, and, more recently, mammalian siderophores have been identified (Bao et al., 2010; Devireddy et al., 2010). The biological effects of Lcn 2 on mammalian cells depend on its iron status. Lcn 2 loaded with a siderophore/iron complex can deliver iron to cells (Yang et al., 2002; Devireddy et al., 2005), whereas Lcn 2 without bound siderophore or iron has been shown to deplete intracellular iron levels and cause apoptosis (Devireddy et al., 2005). The iron status of Lcn 2 produced as a result of inflammation has not been directly assessed. Increased Lcn2 expression is an innate immune defense mechanism aimed at preventing bacteria from acquiring iron, and, in the injured spinal cord, it is likely an innate immune mechanism to prevent infections after injury. In the absence of any bacterial siderophore, however, it is conceivable that Lcn2 would bind its mammalian receptors and exert its iron-binding effects within host cells. One effect of intracellular iron depletion that has already been mentioned is apoptosis; however, intracellular iron levels have also been shown to regulate a number of other processes, including inflammation (Cheepsunthorn et al., 2001; Wang et al., 2008), cell motility (Zhang et al., 2009), and cell division (Yu et al., 2007).

Alternatively, Lcn2 bound to an unknown endogenous iron-binding protein may serve to transport iron to other cells. Whether this occurs in vivo and in the CNS is still unknown. Recent reports suggest that Lcn2 has bifunctional effects on microglia and astrocytes in vitro (Lee et al., 2007, 2009). The addition of $\operatorname{Lcn} 2$, as well as the forced expression of Lcn 2 in astrocyte cultures, caused an increase in GFAP expression and morphological changes consistent with reactive astrogliosis (Lee et al., 2009). On the other hand, $\mathrm{Lcn} 2$ sensitized astrocytes in vitro to various cytotoxic stimuli, including NO-mediated apoptosis, and promoted cell death. The suppressed expression of Lcn 2 by antisense knockdown in astrocytes promoted cell survival (Lee et al., 2009). In the injured spinal cord, we did not detect Lcn 2 and Lcn $2 \mathrm{R}$ by immunofluorescence labeling in microglia, but high levels were seen in astrocytes. We also observed that the crosssectional area of the spinal cord occupied by GFAP astrocytes was greater in $\mathrm{Lcn} 2^{-1-}$ mice at 3 and 7 dpi than in injured wild-type controls, which may contribute to the increased tissue sparing seen in the knock-out mice. An important protective role for astrocytes in controlling or limiting the inflammatory response at early time points after spinal cord injury has been proposed previously (Okada et al., 2006; Sofroniew, 2009). The contusion injury model that we have used produces significant white and gray matter damage. Our results suggest that the absence of Lcn2 increases tissue sparing as assessed by GFAP staining and neuronal counts. Recombinant Lcn 2 did not induce any loss of viability of primary cerebellar neurons in vitro assayed using the lactate dehydrogenase assay (data not shown), suggesting that the loss of neurons after SCI may be secondary to the inflammatory response. White matter sparing, which is associated with improved recovery from this kind of injury (Magnuson et al., 2005), is also increased in $\mathrm{Lcn} 2^{-1-}$ mice, and these mice show greater sparing of myelin after SCI. Our immunostaining results, however, showed little or no expression of Lcn 2 and its receptor in oligodendrocytes, suggesting that the increased myelin sparing we observed is likely an indirect effect of Lcn2 deficiency.

Increasing evidence suggests that Lcn2 plays an important role in the innate immune response, primarily as a defense against 

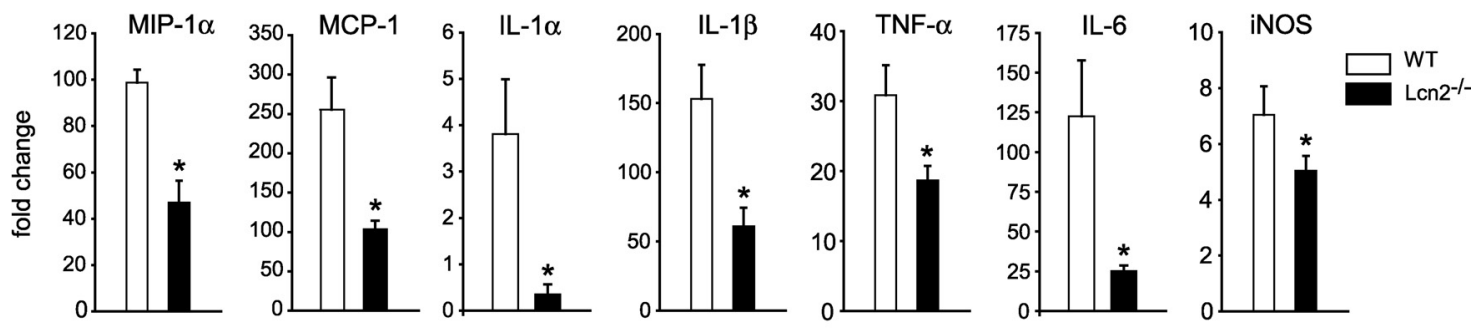

1 day post injury

Figure 6. Reduced expression of pro-inflammatory mediators in LCn2 ${ }^{-1-}$ mice. The bar graphs show the relative mRNA expression of several pro-inflammatory mediators (MCP-1 $\alpha$, MCP-1, IL- $1 \alpha$, IL-1 $\beta$, TNF- $\alpha$, IL-6, and iNOS) in the spinal cord at $1 \mathrm{dpi}$ as determined by QRT-PCR. The expression of all of these mediators is significantly reduced in $L \mathrm{cn} 2^{-1-}$ mice. Data are shown as mean \pm SEM. ${ }^{*} p<0.05$, two-tailed $t$ test, $n=4$ each genotype.

bacterial infection. However, our results suggest that, in the setting of aseptic SCI, Lcn2 may also have significant detrimental effects. We provide evidence that Lcn2 and its receptor are expressed after SCI, and that $\mathrm{Lcn} 2$ plays a detrimental role in SCI, contributing to neuronal and astrocyte loss, pro-inflammatory cytokine expression, and immune cell infiltration. Our results have implications for other inflammatory CNS conditions that are characterized by high levels of Lcn2 expression.

\section{References}

Bao G, Clifton M, Hoette TM, Mori K, Deng SX, Qiu A, Viltard M, Williams D, Paragas N, Leete T, Kulkarni R, Li X, Lee B, Kalandadze A, Ratner AJ, Pizarro JC, Schmidt-Ott KM, Landry DW, Raymond KN, Strong RK, et al. (2010) Iron traffics in circulation bound to a siderocalin (Ngal)-catechol complex. Nat Chem Biol 6:602-609.

Bartholdi D, Schwab ME (1997) Expression of pro-inflammatory cytokine and chemokine mRNA upon experimental spinal cord injury in mouse: an in situ hybridization study. Eur J Neurosci 9:1422-1438.

Basso DM, Fisher LC, Anderson AJ, Jakeman LB, McTigue DM, Popovich PG (2006) Basso Mouse Scale for locomotion detects differences in recovery after spinal cord injury in five common mouse strains. J Neurotrauma 23:635-659.

Berger T, Cheung CC, Elia AJ, Mak TW (2010) Disruption of the Lcn2 gene in mice suppresses primary mammary tumor formation but does not decrease lung metastasis. Proc Natl Acad Sci U S A 107:2995-3000.

Bolignano D, Coppolino G, Lacquaniti A, Buemi M (2010) From kidney to cardiovascular diseases: NGAL as a biomarker beyond the confines of nephrology. Eur J Clin Invest 40:273-276.

Chan YR, Liu JS, Pociask DA, Zheng M, Mietzner TA, Berger T, Mak TW, Clifton MC, Strong RK, Ray P, Kolls JK (2009) Lipocalin 2 is required for pulmonary host defense against Klebsiella infection. J Immunol 182:4947-4956.

Cheepsunthorn P, Radov L, Menzies S, Reid J, Connor JR (2001) Characterization of a novel brain-derived microglial cell line isolated from neonatal rat brain. Glia 35:53-62.

De Biase A, Knoblach SM, Di Giovanni S, Fan C, Molon A, Hoffman EP, Faden AI (2005) Gene expression profiling of experimental traumatic spinal cord injury as a function of distance from impact site and injury severity. Physiol Genomics 22:368-381.

Devireddy LR, Gazin C, Zhu X, Green MR (2005) A cell-surface receptor for lipocalin $24 \mathrm{p} 3$ selectively mediates apoptosis and iron uptake. Cell 123:1293-1305. SEM).
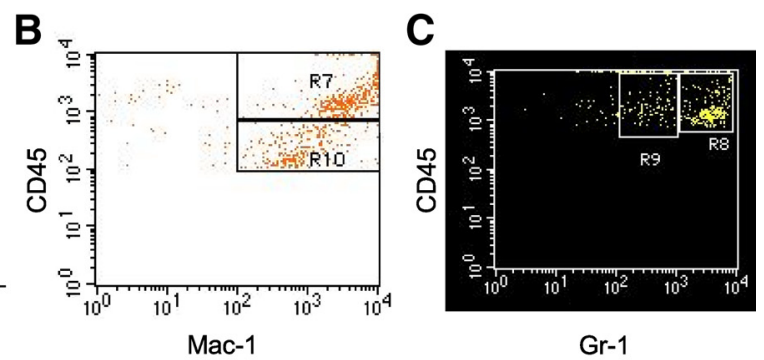

E
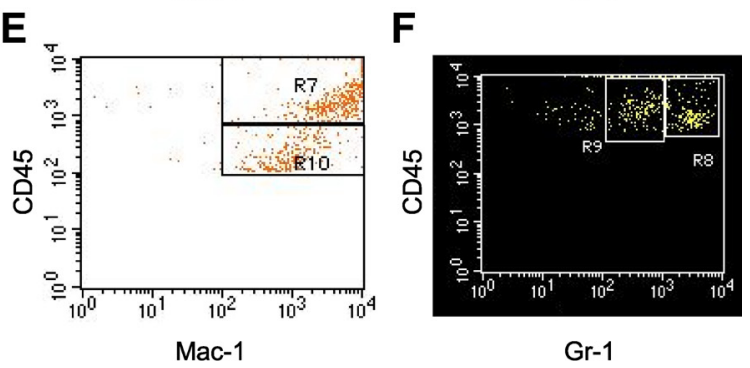

Figure 7. Altered numbers of neutrophils and monocytes in the injured spinal cord in $L \mathrm{Cn} 2^{-/-}$mice. Representative density plots of fluorescence-activated cell sorting analysis of cells isolated from injured spinal cord at 2 dpi. All CD $45^{+}$cells $(A, D, R 5)$ were further gated on Mac- 1 labeling $(\boldsymbol{B}, \boldsymbol{E})$. In $\boldsymbol{B}$ and $\boldsymbol{E}$, two distinct populations of $\mathrm{Mac}^{+}{ }^{+}$cells can be observed, i.e., the $\mathrm{CD} 45^{\text {high }}$ (box (box labeled R10) populations. The CD45 ${ }^{\text {high }}$ population are a mixed population of blood-derived population (R7) was further gated on Gr-1 labeling $(\boldsymbol{C}, \boldsymbol{F})$. Two $\mathrm{Gr}-1^{+}$populations emerge, $\mathrm{Gr}-1^{\text {high }}$ (box labeled R8) and $\mathrm{Gr}-1^{\text {low }}$ There is a significantly smaller Gr-1 ${ }^{\text {high }}$ population in $L\left(n 2^{-/-}\right.$injured spinal cords and a significantly larger $\mathrm{Gr}-1^{\text {low }}$ population in ${ }^{-1-}$ injured spinal cord than in wild-type controls $[p<0.05,2$-tailed $t$ test, $n=6$ for wild-type (WT), $n=5$ for knock-out (KO)]. Gr- $1^{\text {high }}$ in WT $=59.5 \pm 0.86 \%$, in KO $=49.6 \pm 0.97 \% . G r-1^{\text {low }}$ in WT $=25.1 \pm 0.51 \%$, in K0 $=30.3 \pm 0.82 \%$ (mean \pm

Devireddy LR, Hart DO, Goetz DH, Green MR (2010) A mammalian siderophore synthesized by an enzyme with a bacterial homolog involved in enterobactin production. Cell 141:1006-1017.

Flo TH, Smith KD, Sato S, Rodriguez DJ, Holmes MA, Strong RK, Akira S, Aderem A (2004) Lipocalin 2 mediates an innate immune response to bacterial infection by sequestrating iron. Nature 432:917-921.

Ghasemlou N, Kerr BJ, David S (2005) Tissue displacement and impact force are important contributors to outcome after spinal cord contusion injury. Exp Neurol 196:9-17.

Glezer I, Chernomoretz A, David S, Plante MM, Rivest S (2007) Genes involved in the balance between neuronal survival and death during inflammation. PLoS ONE 2:e310.

Goetz DH, Holmes MA, Borregaard N, Bluhm ME, Raymond KN, Strong RK (2002) The neutrophil lipocalin NGAL is a bacteriostatic agent that interferes with siderophore-mediated iron acquisition. Mol Cell 10:1033-1043.

Gris D, Marsh DR, Oatway MA, Chen Y, Hamilton EF, Dekaban GA, Weaver LC (2004) Transient blockade of the CD11d/CD18 integrin reduces secondary damage after spinal cord injury, improving sensory, autonomic, and motor function. J Neurosci 24:4043-4051.

Huang H, Akira S, Santos MM (2009) Is the iron donor lipocalin 2 impli- 
cated in the pathophysiology of hereditary hemochromatosis? Hepatology 49:1012-1016.

Kjeldsen L, Cowland JB, Borregaard N (2000) Human neutrophil gelatinaseassociated lipocalin and homologous proteins in rat and mouse. Biochim Biophys Acta 1482:272-283.

Lee S, Lee J, Kim S, Park JY, Lee WH, Mori K, Kim SH, Kim IK, Suk K (2007) A dual role of lipocalin 2 in the apoptosis and deramification of activated microglia. J Immunol 179:3231-3241.

Lee S, Park JY, Lee WH, Kim H, Park HC, Mori K, Suk K (2009) Lipocalin-2 is an autocrine mediator of reactive astrocytosis. J Neurosci 29:234-249.

Livak KJ, Schmittgen TD (2001) Analysis of relative gene expression data using real-time quantitative PCR and the 2(-Delta Delta C(T)) Method. Methods 25:402-408.

Magnuson DS, Lovett R, Coffee C, Gray R, Han Y, Zhang YP, Burke DA (2005) Functional consequences of lumbar spinal cord contusion injuries in the adult rat. J Neurotrauma 22:529-543.

Marques F, Rodrigues AJ, Sousa JC, Coppola G, Geschwind DH, Sousa N, Correia-Neves M, Palha JA (2008) Lipocalin 2 is a choroid plexus acutephase protein. J Cereb Blood Flow Metab 28:450-455.

Miharada K, Hiroyama T, Sudo K, Nagasawa T, Nakamura Y (2005) Lipocalin 2 functions as a negative regulator of red blood cell production in an autocrine fashion. FASEB J 19:1881-1883.

Nairz M, Theurl I, Schroll A, Theurl M, Fritsche G, Lindner E, Seifert M, Crouch ML, Hantke K, Akira S, Fang FC, Weiss G (2009) Absence of functional Hfe protects mice from invasive Salmonella enterica Serovar Typhimurium infection via induction of lipocalin-2. Blood 114:3642-3651.

Okada S, Nakamura M, Katoh H, Miyao T, Shimazaki T, Ishii K, Yamane J, Yoshimura A, Iwamoto Y, Toyama Y, Okano H (2006) Conditional ablation of Stat 3 or Socs 3 discloses a dual role for reactive astrocytes after spinal cord injury. Nat Med 12:829-834.

Pineau I, Lacroix S (2007) Proinflammatory cytokine synthesis in the injured mouse spinal cord: multiphasic expression pattern and identification of the cell types involved. J Comp Neurol 500:267-285.

Pineau I, Sun L, Bastien D, Lacroix S (2010) Astrocytes initiate inflammation in the injured mouse spinal cord by promoting the entry of neutrophils and inflammatory monocytes in an IL-1 receptor/MyD88-dependent fashion. Brain Behav Immun 24:540-553.

Popovich PG, Longbrake EE (2008) Can the immune system be harnessed to repair the CNS? Nat Rev Neurosci 9:481-493.

Rathore KI, Kerr BJ, Redensek A, López-Vales R, Jeong SY, Ponka P, David S (2008) Ceruloplasmin protects injured spinal cord from iron-mediated oxidative damage. J Neurosci 28:12736-12747.

Saiga H, Nishimura J, Kuwata H, Okuyama M, Matsumoto S, Sato S, Matsumoto M, Akira S, Yoshikai Y, Honda K, Yamamoto M, Takeda K (2008) Lipocalin 2-dependent inhibition of mycobacterial growth in alveolar epithelium. J Immunol 181:8521-8527.

Serhan CN, Chiang N, Van Dyke TE (2008) Resolving inflammation: dual anti-inflammatory and pro-resolution lipid mediators. Nat Rev Immunol 8:349-361.

Shechter R, London A, Varol C, Raposo C, Cusimano M, Yovel G, Rolls A, Mack M, Pluchino S, Martino G, Jung S, Schwartz M (2009) Infiltrating blood-derived macrophages are vital cells playing an anti-inflammatory role in recovery from spinal cord injury in mice. PLoS Med 6:e1000113.

Sofroniew MV (2009) Molecular dissection of reactive astrogliosis and glial scar formation. Trends Neurosci 32:638-647.

Stirling DP, Yong VW (2008) Dynamics of the inflammatory response after murine spinal cord injury revealed by flow cytometry. J Neurosci Res 86:1944-1958.

Stirling DP, Liu S, Kubes P, Yong VW (2009) Depletion of Ly6G/Gr-1 leukocytes after spinal cord injury in mice alters wound healing and worsens neurological outcome. J Neurosci 29:753-764.

Taoka Y, Okajima K, Uchiba M, Murakami K, Kushimoto S, Johno M, Naruo M, Okabe H, Takatsuki K (1997) Role of neutrophils in spinal cord injury in the rat. Neuroscience 79:1177-1182.

Triebel S, Bläser J, Reinke H, Tschesche H (1992) A 25 kDa alpha 2-microglobulin-related protein is a component of the $125 \mathrm{kDa}$ form of human gelatinase. FEBS Lett 314:386-388.

Tveita AA, Rekvig OP, Zykova SN (2008) Increased glomerular matrix metalloproteinase activity in murine lupus nephritis. Kidney Int 74:1150-1158.

Wandersman C, Delepelaire P (2004) Bacterial iron sources: from siderophores to hemophores. Annu Rev Microbiol 58:611-647.

Wang L, Johnson EE, Shi HN, Walker WA, Wessling-Resnick M, Cherayil BJ (2008) Attenuated inflammatory responses in hemochromatosis reveal a role for iron in the regulation of macrophage cytokine translation. J Immunol 181:2723-2731.

Yang J, Goetz D, Li JY, Wang W, Mori K, Setlik D, Du T, Erdjument-Bromage H, Tempst P, Strong R, Barasch J (2001) An iron delivery pathway mediated by a lipocalin. Mol Cell 10:1045-1056.

Yang L, Blumbergs PC, Jones NR, Manavis J, Sarvestani GT, Ghabriel MN (2004) Early expression and cellular localization of proinflammatory cytokines interleukin-1beta, interleukin-6, and tumor necrosis factor-alpha in human traumatic spinal cord injury. Spine 29:966-971.

Yang L, Jones NR, Blumbergs PC, Van Den Heuvel C, Moore EJ, Manavis J, Sarvestani GT, Ghabriel MN (2005) Severity-dependent expression of pro-inflammatory cytokines in traumatic spinal cord injury in the rat. J Clin Neurosci 12:276-284.

Yu Y, Kovacevic Z, Richardson DR (2007) Tuning cell cycle regulation with an iron key. Cell Cycle 6:1982-1994.

Zhang KH, Tian HY, Gao X, Lei WW, Hu Y, Wang DM, Pan XC, Yu ML, Xu GJ, Zhao FK, Song JG (2009) Ferritin heavy chain-mediated iron homeostasis and subsequent increased reactive oxygen species production are essential for epithelial-mesenchymal transition. Cancer Res 69:53405348. 